\title{
RETROPERITONEAL LYMPHADENECTOMY BY VIDEOLAPAROSCOPIC TRANSPERITONEAL APPROACH IN PATIENTS WITH NON- SEMINOMATOUS TESTICULAR TUMOR
}

\author{
M. TOBIAS-MACHADO, JOÃO P. ZAMBON, ALEXANDRE D. FERREIRA, JIMMY A. \\ MEDINA, ROBERTO V. JULIANO, ERIC R. WROCLAWSKI
}

Discipline of Urology, ABC Medicine School, Santo André, São Paulo, Brazil

\begin{abstract}
Objective: The present study aims to report the preliminary experience with videolaparoscopic retroperitoneal lymphadenectomy in the treatment of patients with non-seminomatous testicular tumor.

Materials and Methods: Seven surgeries were performed in order to access retroperitoneal lymph nodes in patients with non-seminomatous testicular cancer. We performed the videolaparoscopic retroperitoneal lymphadenectomy (LRL) technique in 5 patients with stage I disease and laparoscopic resection of residual mass (LRRM), following chemotherapy (ChT), in 2 patients with stage II disease. Initial approach was obtained through 4 trocars, using an incision in supra-umbilical midline when manual assistance was required. Surgical time was analyzed, as well as blood loss, need for analgesic drugs postoperatively, hospital stay, complications, need for blood transfusion, histopathological data and tumor control in a mean follow-up of 18 months.

Results: Mean surgical time was 200 to 260 minutes in LRL and LRRM groups respectively, mean blood loss was $300 \mathrm{~mL}$ for the LRL group and $400 \mathrm{~mL}$ for the LRRM group, without need for transfusions. There was a lesion in the vena cava in the LRL group, which was managed with manual assistance and one conversion in the LRRM group, due to a 10-cm tumor mass that was adhered to the aorta. Mean hospital stay was 3 days, excluding the converted case, and the use of analgesic drugs was needed until the second postoperative day. Of the stage I patients, 2 had active disease in retroperitoneum, and underwent adjuvant ChT. The 2 residual masses were teratomas. There was no recurrence during the follow-up period.

Conclusions: Videolaparoscopic retroperitoneal lymphadenectomy is a procedure with high technical complexity and a higher potential for conversion when performed following chemotherapy.
\end{abstract}

Key words: testis; testicular neoplasms; germ cell tumors; chemotherapy; lymphadenectomy; laparoscopy

Int Braz J Urol. 2004; 30: 389-97

\section{INTRODUCTION}

Conventional retroperitoneal lymphadenectomy is a major surgical procedure, which confers considerable morbidity to patients with nonseminomatous testicular tumor. In patients with advanced disease who are already debilitated by the effects of chemotherapy, the surgical procedure will bring an additional morbidity, impairing their quality of life (1).

The retroperitoneal lymphadenectomy is indicated mainly for staging of stage I nonseminomatous tumors, and the resection of residual masses following chemotherapy (ChT) (1). The laparoscopic surgery has lower morbidity when compared with open surgery and some studies show that 
there is no impairment in the oncologic control of the disease (2-12).

Pathological staging of retroperitoneal lymph nodes in patients with non-seminomatous tumors offers 2 advantages: patients with metastatic disease can be identified and treated, and those without metastatic disease can be closely observed $(1,11,12)$.

In patients with metastatic testicular tumors who undergo previous ChT, there is formation of cicatricial tissue that adheres to the great vessels, which can make tumor resection difficult following ChT $(5,8)$. The usual chemotherapy scheme using cisplatin as basic drug provides 60 to $70 \%$ of satisfactory results, while approximately $30 \%$ of patients should undergo a surgical procedure $(11,12)$. In such cases, it is recommended that the laparoscopic surgery as a minimally invasive procedure should be performed with caution, due to the risk of vascular damage with consequent conversion to open surgery, increasing postoperative morbidity $(5,7,8,10-12)$.

In Brazil, the use of the laparoscopic approach has been performed in a few centers, due to the rarity of the disease and the technical complexity of the procedure (8). The objective of this work was to report the preliminary experience with videolaparoscopic retroperitoneal lymphadenectomy in the treatment of patients with non-seminomatous testicular tumor.

\section{MATERIALS AND METHODS}

\section{Selection Criteria}

In the period from January 1999 to December 2002, we retrospectively studied 7 patients diagnosed with non-seminomatous testicular cancer who underwent laparoscopic retroperitoneal lymphadenectomy (Table-1). Patients did not present any contra-indication for surgery and were in good general conditions, with American Society of Anesthesiologists (ASA) score I and II. All patients were operated in a reference teaching institution in Brazil, by the same surgeon.

Laparoscopic retroperitoneal lymphadenectomy was performed in the following situations: 1) Staging for stage I non-seminomatous testicular cancer presenting risks factors such as vascular invasion, invasion of spermatic cord or predominance of embryonal carcinoma in neoplasic components. 2) -
Post-chemotherapy resection of residual masses smaller than $6 \mathrm{~cm}$ as measured by tomography.

The procedure was always initiated by laparoscopic transperitoneal approach. The hand-assisted technique was used as an alternative to avoid conversion to exclusive open surgery in those patients who presented intraoperative complications or in cases where postchemotherapy fibrosis prevented the safe dissection of the great vessels, (13).

Preoperative staging was performed using thorax and abdomen computerized tomography (CT) and tumor markers (alpha-fetoprotein, beta-subunit of human chorionic gonadotropin and lactic dehydrogenase). The employed chemotherapy scheme, if indicated, was 4 PEB cycles (cisplatin, etoposide and bleomycin).

Medical records were assessed in order to observe technical aspects, postoperative complication, conversion rate and postoperative outcome in relation to tumor control. The clinical follow-up of patients ranged from 10 to 28 months.

Five patients had their surgeries indicated for tumoral staging, with 3 cases due to the presence of a predominant embryonal component and 2 due to vascular invasion.

Among these, 3 patients had tumor in the right testis and 2 in the left one. Two patients underwent postchemotherapy resection of residual retroperitoneal mass.

Table 1 - General demographic data of patients who underwent laparoscopic retroperitoneal lymphadenectomy (LRL) for staging and laparoscopic resection of residual mass (LRRM).

\begin{tabular}{lcc}
\hline & LRL & LRRM \\
\hline Number of patients & 5 & 2 \\
Age (years) & 25 & 27 \\
Right-sided tumor & 3 & - \\
Left-sized tumor & 2 & 2 \\
Tumor size on tomography & - & $5-6 \mathrm{~cm}$ \\
Body mass index & 23 & 21 \\
Asa I & 3 & - \\
Asa II & 2 & 2 \\
Stage I & 5 & - \\
Stage IIa & - & 2 \\
\hline
\end{tabular}




\section{Transperitoneal Laparoscopic Retroperito- neal Lymphadenectomy}

\section{Surgical Technique}

1 - Preoperative: Patients were admitted to hospital one day prior to surgery and underwent 8-hour fasting and reservation of red cells concentrate. All underwent general anesthesia with vesical catheter, nasogastric tube and antibiotic coverage during anesthetic induction with first generation cephalosporin. Ureteral catheterization, to facilitate intraoperative identification of ureters, was not performed in any case.

2 - Positioning and installation of trocars: After positioning the patient in lateral decubitus at 60 degrees, 4 trocars were placed: one $10-\mathrm{mm}$ trocar in the umbilical scar, for introducing the 0-degree optics, two 5-mm trocars, one in the midline between the umbilical scar and the xiphoid process and the other in the midline between the pubis and the umbilical scar. This set-up allows the port incisions to be united inside the surgical incision, in case of conversion to open surgery. A 10-mm trocar was placed $2 \mathrm{~cm}$ below the umbilical scar at the lateral margin of the rectus muscle of abdomen on the side to be approached (Figure-1).

3 - Dissection technique: The dissection limits were the same as in open surgery (7). Access to the retroperitoneal space was achieved by an incision in the Toldt line, anterior and medially displacing the colon. For the 5 patients with stage I tumor, the modified lymphadenectomy was performed with interaortocaval dissection. For right-sided testicular tumors, the upper dissection limits were the renal hilum bilaterally, including the ureter at the left and extending downwards until the inferior mesenteric artery. At this point, the dissection was directed to the right side, following the right aortic margin and the right common iliac artery until the crossing point of the ureter. Posterior dissection limit corresponded to the anterior spinous ligament. For tumors located in the left testis, the dissection was similar to the previous one, being more economic for the contralateral side, where the inferior limit was the inferior vena cava and not the ureter.

Ultrasonic or bipolar scalpel was used for resecting the lymphatic tissue and the surgical speci-

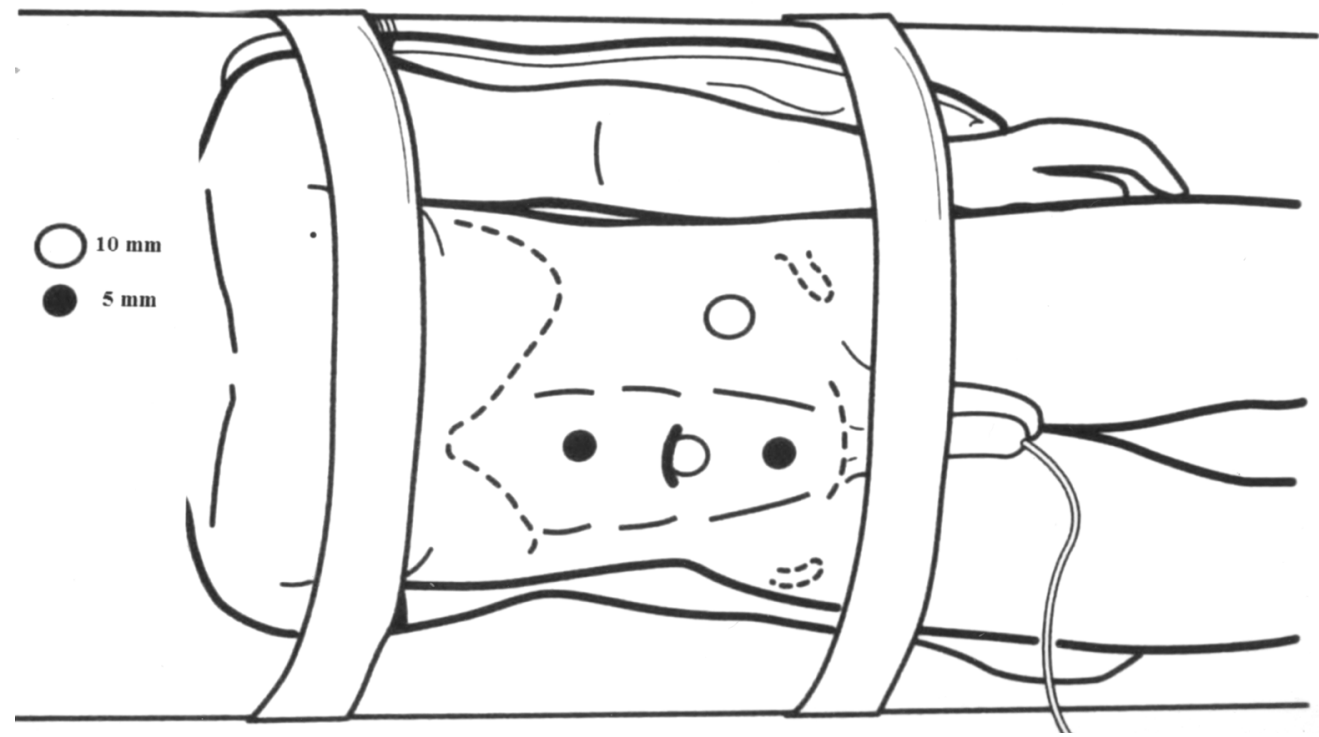

Figure 1 - Positioning and trocars in pure technique for laparoscopic retroperitoneal lymphadenectomy. 


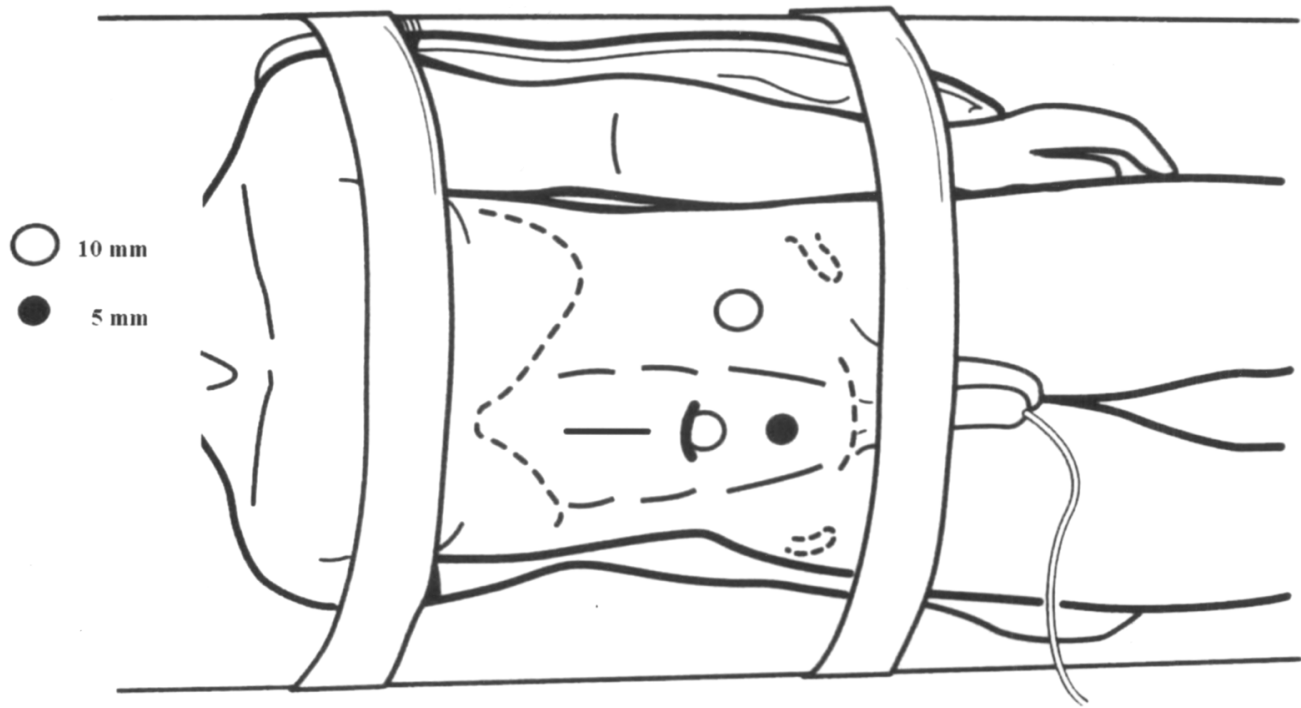

Figure 2 - Positioning, trocars and incision in hand-assisted technique for laparoscopic retroperitoneal lymphadenectomy.

men was removed through entrapment in plastic packaging.

\section{Hand-Assisted Retroperitoneal Lymphadenectomy}

\section{Surgical Technique}

This technique was performed through a midline supra-umbilical incision located above the renal hilum or close to the lesion, without using manual device, including whenever possible the location of one of the previously described ports (Figures-2 and 3).

In the 2 patients with post-ChT residual mass, total resection of the mass was performed.

\section{RESULTS}

In the group of patients with surgical indication for staging, mean age was 25 years and mean body mass index was $23 \mathrm{~kg} / \mathrm{m}^{2}$. Mean surgical time was 200 minutes (160 - 360) and mean blood loss was $300 \mathrm{~mL}$, with no need for transfusion.

There was one intra-operative complication with damage to the inferior vena cava, which was resolved by vascular control, using hand-assisted access, with a 7-mm supra-umbilical midline incision, including the supra-umbilical port, without a device for pneumoperitoneum contention. After digital compression of the vena cava, it was possible to place 2 Doyan valves, applying a Satinski clamp and closing the lesion with 5-0 Prolene suture, externally, with no additional enlargement of the incision. In this case there was no impairment in relation to the laparoscopic access, since, once the vascular suture was completed, we were able to conclude the procedure through hand-assisted laparoscopic approach. Thus, we did not consider this temporary transition between open and laparoscopic techniques as a conversion to definitive open approach. There was no conversion to definitive open surgery in this group.

In the LRRM group, mean age was 27 years and mean body mass index was $21 \mathrm{~kg} / \mathrm{m} 2$. Mean surgical time was 260 minutes (240-280) and mean blood loss was $400 \mathrm{~mL}$, and blood transfusions were not required. There was no intraoperative complication, and in one case, we needed to perform a conversion to open surgery due to an extensive tumor, larger than $10 \mathrm{~cm}$, located in para- and retro-aortic regions. Pre- 
Table 2 - Intraoperative data from LRL and LRRM.

\begin{tabular}{lcc}
\hline & LRL & LRRM \\
\hline Surgical time (min) & $200(160-360)$ & $260(240-280)$ \\
Blood loss (mL) & 300 & 400 \\
Transfusion & 0 & 0 \\
Intraoperative & $1(12 \%)$ & 0 \\
$\quad$ complications & & 1 \\
Conversion & 0 & 1 \\
\hline
\end{tabular}

Table 3 - Postoperative data from LRL and LRRM.

\begin{tabular}{lll}
\hline & LRL & LRRM \\
\hline Hospital stay (days) & $3(1-4)$ & $3(2-4)$ \\
Analgesic use (days) & $2(1-3)$ & $2.5(2-3)$ \\
Time to oral restitution (days) & $2(1-3)$ & $2(1-3)$ \\
\hline
\end{tabular}

operative tomography performed 45 days before the surgery revealed a $6-\mathrm{cm}$ residual mass, and for this reason, the patient was included in the study (Table-2).

Data relative to postoperative outcome can be observed in Table-3.

No postoperative complication was observed during the follow-up, in the 2 groups of patients.

Of the 5 stage I patients, 2 presented positive lymph nodes for non-seminomatous tumor and subsequently underwent adjuvant chemotherapy. In the 2 patients undergoing post-ChT resection of residual mass (Figure-4) the pathological report revealed that it was a teratoma, with no need for complementary treatment.

There was no case of local or systemic recurrence during a mean follow-up of 18 months in both groups under study. Postoperative follow-up revealed that all patients maintained normal anterograde ejaculation.

\section{COMMENTS}

There is much discussion regarding the best approach to stage I non-seminomatous testicular tumors. While some authors advocate a careful followup, based on the efficacy of the chemotherapic drugs used for treating this disease (12), others prefer the retroperitoneal lymphadenectomy with diagnostic and therapeutic purposes. The main argument for this management is the fact that microscopic metastases can be present in approximately $30 \%$ of cases (1216), with $70 \%$ being free from disease without requiring cytotoxic chemotherapy.

Another indication for lymphadenectomy is related to the assessment of post-ChT response in patients with residual tumors in advanced stage. With the availability of effective second-line chemotherapic agents, a proper diagnosis can be fundamental for an

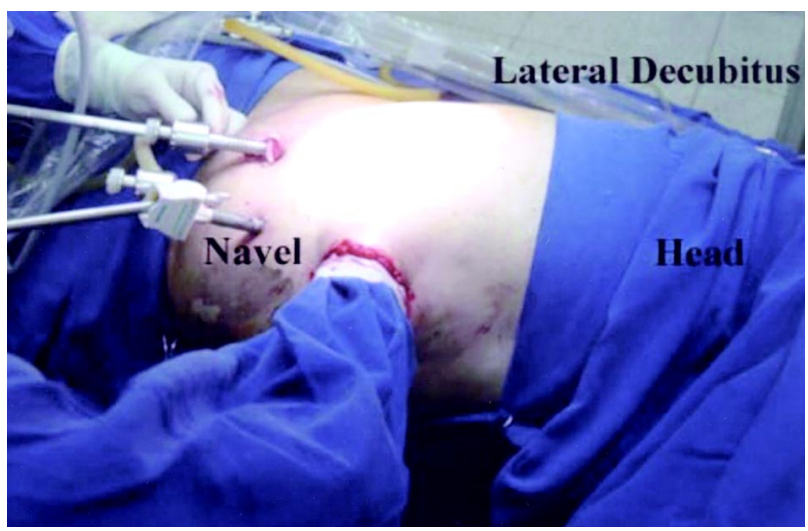

Figure 3 - Positioning, trocars and surgical team in hand-assisted technique for laparoscopic retroperitoneal lymphadenectomy.

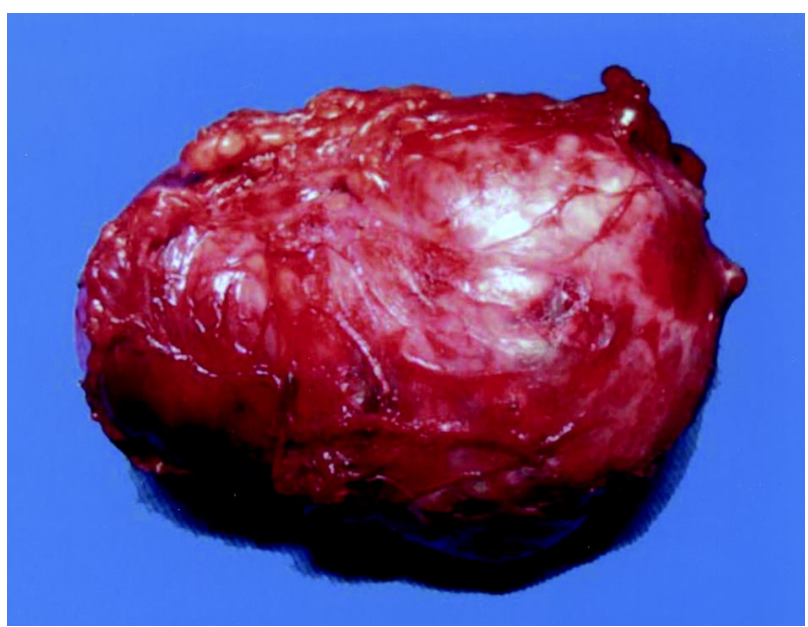

Figure 4 - Post-chemotherapy retroperitoneal residual mass. Surgical specimen. 
accurate indication of rescue $\mathrm{ChT}$ in cases with an active malignant component (8-10,12,14-18).

The incidence of retroperitoneal teratoma ranges from 8 to $13 \%(1,8)$. These tumors have a proven malignant potential, grow rapidly, and can invade adjacent structures with consequent functional impairment to the patient. Moreover, these tumors are resistant to radio and chemotherapy $(1,9)$. Considering this evidence, some authors advocate the performance of retroperitoneal lymphadenectomy even in patients with negative radiological findings $(5,9,18)$. In cases with retroperitoneal mass larger than $1.5 \mathrm{~cm}$ or primary tumors up to $5 \mathrm{~cm}$, regardless the response to ChT, since they present negative tumoral markers, lymphadenectomy has a precise indication $(5,8-10,12)$. In the present study, the 2 post-ChT residual masses were teratomas and the surgery certainly benefited the patients $(2,3,10,12)$.

Especially in laparoscopic access, where there is greater technical difficulty for lymphatic resection posterior to the great vessels, it is currently recommended to perform the modified unilateral resection or isolated resection of the post-ChT residual mass. This proposal is based on the distribution of retroperitoneal metastases for nonseminomatous tumors proposed by Wood et al. (11). According to these authors, the lymphatic drainage on the right side is directed to the lymph nodes located in the interaortocaval space, and to the left para-aortic and pre-aortic lymph nodes on the left side. In patients who underwent previous ChT, the sites of metastatic spread are similar to the sites of primary tumor and approximately $8 \%$ of the tumors would be outside the resection area $(11,12)$. Previously described data suggest that there is no benefit in routine retroaortic and retrocava lymphadenectomy. In this study, we performed retrocava dissection only in our first patient (case with longer surgical time). Routinely, we adopted the interaortocaval dissection regardless the side where the primary tumor was located, since the possibility of metastasis at this site must be taken into account. We did not observe local or systemic recurrence with the reported technique after a mean follow-up of 18 months.
Using the unilateral dissection below the inferior mesenteric artery (modified lymphadenectomy), approximately 85 to $90 \%$ of patients have their ejaculatory function preserved $(1-3,6,9,14)$. In this small sample, all patients maintained preserved antegrade ejaculation. This is fundamentally important, since the main age range affected by testicular neoplasia comprises young adults in active reproductive phase. In our initial patient sample, we observed surgical and outcome results very similar to other works in the literature $(2,4,6,14)$.

As previously exposed, the videolaparoscopic technique that we employed is similar to open surgery. For releasing the lymphatic tissue, we preferred to use the ultra-sonic scalpel, because it reduces the risk of thermal damage to the great vessels and adjacent organs.

Chemotherapy previously to surgery can make laparoscopic dissection difficult, due to the occurrence of local fibrosis of the retroperitoneal tissue that becomes closely adhered to vascular structures, with higher conversion indexes. However, previous ChT per se do not prevent the resection of post-ChT mass by videolaparoscopic approach $(5,8,10,12)$. Some works are contrary to resecting the post-chemotherapy residual mass by videolaparoscopic access (9). Others advocate laparoscopic surgery for masses measuring up to 5 $\mathrm{cm}$, showing that this is feasible, with a higher conversion index $(5,8,10,12)$.

Sutherland \& Wright reported one case of resection of a thoracoabdominal mass successfully using thoracoscopy and hand-assisted laparoscopic access (13).

The manual conversion in cases of emergency or difficulty for dissection is original and has not been described in previously published works. We believe that using this maneuver may be extremely useful, especially when the surgeon is in the learning curve or when vascular laparoscopic material is not available. Greater skills and technical ability with vascular sutures by laparoscopic approach make this maneuver unnecessary. Even when using a 6- to 7-mm incision, including the orifice of one of the ports, it is possible to preserve the postoperative benefits of a minimally invasive surgery (13). 
There are few works reporting the results of long-term oncologic control (19). Additionally, in series of laparoscopic surgery, patients presenting positive lymph nodes systematically receive adjuvant chemotherapy, thus it is not possible to assess surgical results separately. Anyway, the oncologic control seems to be similar to the open technique $(2-12,19)$.

\section{CONCLUSIONS}

Videolaparoscopic retroperitoneal lymphadenectomy is a procedure with high technical complexity and a higher potential for emergency conversion when performed after chemotherapy. In the absence of laparoscopic vascular material, a manual incision can be sufficient to avoid conversion, maintaining the advantages of the minimally invasive surgery.

We believe that this method is feasible for diagnosing and treating stage I and IIa nonseminomatous tumors, or when the postchemotherapy residual mass is smaller than $6 \mathrm{~cm}$.

\section{REFERENCES}

1. Richie JP, Steele GS: Neoplasm of the Testis. In: Walsh PC, Retik AB, Vaughan ED, Wein JA (eds). Campbell's Urology, ed. 8. Philadelphia, WB Saunders. 2002; pp. 2876-919.

2. Giusti G, Beltrami P, Tallarigo C, Bianchi G, Mobilio G: Unilateral laparoscopic retroperitoneal lymphadenectomy for clinical stage I non-seminomatous testicular cancer. J Endourol. 1998; 12: 561-6.

3. Janetschek G, Hobisch A, Peschel R, Hittmair A, Bartsch G: Laparoscopic retroperitoneal lymph node dissection for clinical stage I non-seminomatous testicular carcinoma: long-term outcome. J Urol. 2000; 163: 1793-6.

4. Rassweiler JJ, Frede T, Lenz E, Seemann O, Alken P: Long-term experience with laparoscopic retroperitoneal lymph node dissection in the management of lowstage testis cancer. Eur Urol. 2000; 37: 251-60.

5. Janetschek G, Hobisch A, Hittmnair A, Holti L, Peschel R, Bartsch G: Laparoscopic retroperitoneal lymphadenectomy after chemotherapy for stage II B nonseminomatous testicular carcinoma. J Urol. 1999; 161: 477-81.
6. Janetschek G: Laparoscopic retroperitoneal lymph node dissection. In Sakti Das (ed). The Urologic Clinics of North America-Advanced Urologic Laparoscopy. Philadelphia, WB Saunders. 2001; 28: 107-14.

7. Janetschek G, Hobisch A, Holtl L, Bartsch G: Retroperitoneal lymphadenectomy for clinical stage I nonseminomtous testicular tumor: laparoscopy versus open surgery and impact of learning curve. J Urol. 1996; 156: 89-93.

8. Mariano MB, Tefilli MV: Laparoscopy retroperitoneal lymphadenectomy after chemotherapy for stage II B testicular tumors. Int Braz J Urol. 2001; 27: 527-34.

9. Rassweiler JJ, Seemann O, Henkel TO, Stock C, Frede T, Alken P: Laparoscopic retroperitoneal lymph node dissection for non-seminomatous germ cell tumor: indications and limitations. J Urol. 1996; 156: 1108-13.

10. Rabbani F, Goldenberg SL, Gleave ME, Paterson RF, Murray N, Sullivan LD: Retroperitoneal lymphadenectomy for post-chemotherapy residual masses: is a modified dissection and resection of residual masses sufficient? Br J Urol. 1998; 81: 295-99.

11. Wood DP Jr, Herr HW, Heller G, Vlamis V, Sogani PC, Motzer RJ, et al.: Distribution of retroperitoneal metastasis after chemotherapy in patients with nonseminomatous germ cell tumors. J Urol. 1992; 148: 1812-15.

12. Gelderman WA, Koops HS, Sleijfer DT, Oosterhuis JW, Oldhoff J: Treatment of retroperitoneal residual tumor after PVB chemotherapy of non-seminomatous testicular tumors. Cancer.1986; 58: 1418-21.

13. Sutherland A, Wright G: Hand-assisted laparoscopic lymphadenectomy: a novel approach to a difficult area. ANZ J Surg. 2003; 73: 755-7.

14. Janetschek G: Laparoscopic retroperitoneal lymph node dissection. Urol Clin North Am. 2001; 28: 10714.

15. Donohue JP, Thornhill JA, Foster RS, Rowland RG, Bihrle R: Retroperitoneal lymphadenectomy for clinical stage A non-seminomatous germ cell testis cancer: Review of the Indiana University experience 19651989. Br J Urol. 1993: 71: 326-35.

16. Atsu N, Eskicorapci S, Uner A, Ekici S, Gungen Y, Erkan I, et al.: A novel surveillance protocol for stage I non-seminomatous germ cell testicular tumors. BJU Int. 2003; 92: 32-5.

17. Hoskin P, Dilly S, Easton D, Horwich A, Hendry W, Peckham MJ: Prognostic factors in stage I nonseminomatous germ-cell testicular tumors managed by orchiectomy and surveillance: Implications for adjuvant chemotherapy. J Clin Oncol. 1986; 4: 1031-6. 
18. Bohlen D, Borner M, Sonntag RW, Fey MF, Studer UE: Long-term results following adjuvant chemotherapy in patients with clinical stage I testicular nonseminomatous malignant germ cell tumors with high risk factors. J Urol. 1999; 161: 1148-52.
19. Bhayani SB, Ong A, Oh WK, Kantoff PW, Kavoussi LR: Laparoscopic retroperitoneal lymph node dissection for clinical stage I nonseminimatous germ cell testicular cancer: a long- term update. Urology. 2003; 62: 324-7.
Received: December 23, 2003

Accepted after revision: September 11, 2004

\author{
Correspondence address: \\ Dr. Marcos Tobias-Machado \\ Rua Graúna 104 / 131 \\ São Paulo, SP, 04514-000, Brazil \\ Fax: + 5511 288-1003 \\ E-mail: tobias-machado@uol.com.br
}

\section{EDITORIAL COMMENT}

The authors describe their initial experience with transperitoneal laparoscopic retroperitoneal lymphadenectomy in a group of 7 patients with nonseminomatous testicular tumors. Five patients presented clinical stage I, and 2 patients had postchemotherapy residual masses.

Testicular cancer, though relatively rare, is the most common tumor in men aged between 15 and 35 years. It also represents the solid neoplasia with higher possibility of cure, serving as an example of an almost perfect therapeutic synergism between the different oncologic expertise fields. The dramatic improvement in survival of this population results from the combination of more accurate diagnostic techniques, availability of tumor markers, effective chemotherapy schemes and modifications in surgical techniques, which, jointly, reduced the mortality from $60 \%$ in the 70 s to less than $10 \%$ in mid-90s. Thus, with the availability of effective therapeutic options, even for patients with advanced disease, efforts have been focused to reducing the morbidity, with potential improvements in the current protocols.

In this setting, videolaparoscopic surgery seems to be an attractive approach, both for initial staging of non-seminomatous tumors and for selected cases of patients with postchemotherapy residual masses.

As the authors emphasize, there is a longlasting discussion in urologic literature concerning the best approach to stage I non-seminomatous tumors. While some advocate a careful follow-up, based on the efficacy of chemotherapic agents, others prefer the retroperitoneal lymphadenectomy with diagnostic and therapeutic purposes, stressing the fact that microscopic metastases can coexist in up to $30 \%$ of cases. The laparoscopic retroperitoneal lymphadenectomy appears to provide an optimal alternative in such cases, being a procedure with low morbidity that is routinely used, which allows us to be more liberal when indicating lymphadenectomy in these cases, with better acceptance by patients and clinical oncologists, thus avoiding the anxiety involved in long and consuming observation protocols.

An equally polemic discussion concerns the recommendation of lymphadenectomy in postchemotherapy residual masses. Advanced testicular neoplasias are better managed by a combination of chemotherapy and surgery, conceptually including primary chemotherapy followed by retroperito- 
neal lymphadenectomy for residual masses. In order to reduce the procedural morbidity, we have replaced open classic lymphadenectomy for the laparoscopic approach in selected cases, as it seems to be the tendency of this manuscript's authors. Despite being technically feasible following chemotherapy, we indicate this procedure only to single and/or unilateral multiple residual masses measuring no more than 5 $\mathrm{cm}$. Postchemotherapy laparoscopic retroperitoneal lymphadenectomy is a technically complex procedure with some potentially serious complications, and to the moment it must not be encouraged outside services with a large experience in laparoscopic retroperitoneal surgery.

Dr. Mirandolino B. Mariano Dr. Marcos V. Tefelli Urology Division Mãe de Deus Hospital Porto Alegre, RS, Brazil 